Bhatter College Journal of Multidisciplinary Studies | ISSN 2249-3301, Vol. VII, Number 2, 2017

A Refereed Journal, approved by the UGC \& included in Google Scholar

Article url: www.bcjms.bhattercollege.ac.in/v7/n2/v7n2bnl01.pdf

Article DOI: 10.25274/bcjms.v7n2.v7n2bn101

\title{
তিতাস একটি নদীর নাম' : ব্যক্তিজীবন বনাম গোষ্ঠীজীবনের প্রত্যাশা ও প্রাপ্তির দ্বন্দ্ব
}

চন্দন কুমার সাউ

Ph.D Research scholar, Burdwan University. Email: chandanshow2015@gmail.com

\begin{abstract}
:
বাংলা সাহিত্যে কোন একটি বিশেষ জনগোষ্ঠীর জীবন নিয়ে লেখা উপন্যাসের সংখ্যা কম নয়। আর সেই ধারায় অদ্বৈত মল্লবর্মণের 'তিতাস একটি নদীর নাম' উপন্যাসটির কথা বিশেষভবেই উল্লেখের দাবি রাখে। তিতাস নদী থেকে মাছ ধরে জীবিকা নির্বাহ করা একদল জেলে-সম্প্রদায়ের(মালো সমাজ) জীবন নিয়ে উপন্যাসটি লিখেছেন ঔপন্যাসিক। মালোদের জীবনের এক সামগ্রিক চালচিত্রই তুলে ধরা হয়েছে এই উপন্যালে। প্রতিটি মালো নরনারীর ব্যক্তিগত জীবন, তাদের গোষ্ঠী-জীবনের নিজস্ব সংস্কৃতি ও ঐতিহ্য এবং তিতালের উপর নির্ভরশীল নালোদের জীবন ও জীবিকার পুঙ্খানুপুঙ্খ পরিচয়ই ধরা পড়েছে উপন্যাসটির মধ্যে। আর মালোদের জীবনকথা এই উপন্যাসটির নিবিড় পাঠ নিলে আনরা সমগ্র উপন্যাসের মধ্যে একটা বিষণ্নতার ছোঁয়া তথা একটা ট্র্যাজিক সুর ফুটে উঠতে দেখি।যার মূলে রয়েছে জীবনের প্রত্যাশা ও প্রাপ্তির চিরকালীন দ্বন্দ্ব। আমাদের গবেষণার বিষয়ই হল, অদ্বৈত মল্লবর্মণের 'তিতাস একটি নদীর নাম' উপন্যালে কিভাবে জীবনের এই প্রত্যাশা ও প্রাপ্তির দ্বান্দ্বিক রূপটি ধরা পড়েছে, সেটির উপর আলোকপাত করা।এক্ষেত্রে বিষয়টিকে আমরা দুদিক থেকে দেখাবার চেষ্টা করব-----

(ক) প্রতিটি মালো নরনারীর ব্যক্তিগত জীবন প্রসঙ্গে এই প্রত্যাশা-প্রাপ্তির দ্বন্দ্বের ক্ষেত্রটি।

(খ) আঞ্চলিক উপন্যাস হিসাবে একটি জনগোষ্ঠীর নিজস্ব সংস্কৃতি ও জীবিকার সংকটকে ঘিরে গোষ্ঠীজীবনের ক্ষেত্রে এই প্রত্যাশাপ্রাপ্তির দ্বান্দ্বিক রূপটি।
\end{abstract}

সূচক শব্দঃ অদ্বৈত মল্লবর্মণ, তিতাস একটি নদীর নাম, প্রত্যাশা ও প্রাপ্তির দ্বন্দ্ব, ব্যক্তিজীবন, গোষ্ঠীজীবন

জীবনকে নিয়েই সাহিত্যের পথচলা। বহমান জীবন তার সমস্যাবহুল দ্বান্দ্বিক রূপ নিয়ে উঠে আসে উপন্যাসের পাতায়। কিন্ত যদি প্রশ্ন করা হয়, সেই জীবন কাদের? তাহলে বলতেই হয়---তা যেমন উচ্চবিত্তের জীবন, যেমন মধ্যবিত্তের জীবন, তেমনিই তা আবার প্রান্তিক কোন জনগোষ্ঠীর জীবনও। আর এখানেই বাংলা সাহিত্যে আলাদাভাবে গুরুত্ব দাবি করে আঞ্চলিক উপন্যাস; যেখানে ঔপন্যাসিক একটি অঞ্চলের বিশেষ কোন জনগোষ্ঠীর জীবনকে উপন্যাসের বিষয় হিসাবে গ্রহণ করেন। বাংলা সাহিত্যে বিশেষ কোন একটি জনগোষ্ঠীর জীবন নিয়ে লেখা উপন্যাস হিসাবে মানিক বন্দ্যোপাধ্যায়ের 'পদ্মা নদীর মাঝি', তারাশঙ্কর বন্দ্যোপাধ্যায়ের 'হাঁ সুলী বাঁকের উপকথা', সমরেশ বসুর 'গঙ্গা' ইত্যাদির কথা বলা যায়।আর সেই ধারায় আলাদা মাত্রা যোগ করেছে অদ্বৈত মল্লবর্মণের 'তিতাস একটি নদীর নাম' উপন্যাসটি। অদ্বৈত মল্লবর্মণের স্বল্পকালীন জীবনের শ্রেষ্ঠ কীর্তিই হল তাঁর এই উপন্যাস, যা বাংলা সাহিত্যে তাঁর চিরকালীন আসনটিকে সুনির্দিষ্ট করে দিয়েছে।মূলত অবিভক্ত বাংলাদেশের ত্রিপুরা জেলার তিতাস নদীর তীরবর্তী নিম্নবিত্ত জেলে সম্প্রদায়ের জীবনকে নিয়েই উপন্যাসটি লেখা হয়েছে। যে জেলে সম্প্রদায় সম্পর্কে লেখকের বাস্তব অভিজ্ঞতার জগৎটা ছিল অনেক ব্যাপক, 
কারণ অদ্বৈত মল্লবর্মণ নিজে ছিলেন ওই শ্রেণীভুক্ত।যাইহোক, উপন্যাসটির নিবিড় পাঠ নিলে আমরা দেখি, কোন একটি বিশেষ নায়ক চরিত্র সৃষ্টির প্রতি লেখক গুরুত্ব দেননি; বরং সমগ্র মালোজীবনের সামগ্রিক চিত্রটিকেই তিনি আমাদের সামনে হাজির করেছেন। সরোজ বন্দ্যোপাধ্যায় তাই বলেছেন------

"লেখক যেন এখানে কোন ব্যক্তির কাহিনিকে ধারণ করতে চান না। নদীর যেমন বিশেষের প্রতি কোন পক্ষপাত নেই, কাহিনীও তেমনি এখানে বিশেষ কিছুর ঘাটে বাঁধা নয়।"১

বস্তুত এই উপন্যাস মালোদের জীবন পাঁচালী।আর তিতাস তীরবর্তী মালোজীবনের যে ছবি এই উপন্যাসে ধরা পড়েছে, সেখানে মালোদের জীবনের ছোট-খাট সুখ- দুঃখের বাইরে বিভিন্ন চরিত্রের জীবনকথা প্রসঙ্গে বার বার ঔপন্যাসিক দেখিয়েছেন আমাদের জীবনের প্রত্যাশা আর প্রাপ্তির চিরন্তন দ্বন্বটিকে। মানুষ চায় এক আর বাস্তবে হয় যেন আর এক। নিয়তির অঙ্গুলি হেলনে আমাদের জীবনের সবকিছু যেন কেমন ওলট পালট হয়ে যায় চোখের সামনে।যে স্বপ্নকে আমরা ভেতরে ভেতরে লালন করি তা ভেঙে চুরমার হয়ে যায়, যে প্রতীক্ষা নিয়ে অপেক্ষা করে থাকে মন--- তা আর কাঙ্খিত প্রাপ্তিকে ছুঁতে পারেনা।আর তাই প্রত্যাশা আর প্রাপ্তির এই দ্বন্দ্ব জনিত এক করুণ ট্র্যাজিক সুর যেন আমাদের বহমান জীবনের আপাত আনন্দমুখরতার অন্তরালে অনঃসলিলা ফল্তুধারার মতোই সদা বহমান। মানবজীবনের প্রত্যাশা ও প্রাপ্তির সেই চিরকালীন দ্বন্দ্বটিকেই শাশ্বত জীবনভাবনার আলোকে লেখক বিভিন্ন মালো নরনারীর জীবনকে অবলম্বন করে তুলে ধরেছেন উপন্যাসে।অন্যদিকে উপন্যাসটি নিছক ব্যক্তিজীবনের কাহিনি নয়, বরং তা মালোদের গোষ্ঠীজীবনের স্বরূপকেই তুলে ধরেছে। আর শেষ পর্যন্ত উপন্যাসটিতে ব্যক্তি জীবনকে ছাড়িয়ে এই গোষ্ঠী মালোজীবনের সংকটকেই বড় করে দেখিয়েছেন ঔপন্যাসিক।তিতাসের জল শুকিয়ে যাওয়াকে ঘিরে তিতাস নির্ভর মালোসম্প্রদায়ের অস্তিত্বের সমস্যা আর মালোদের নিজস্ব সংস্কৃতির অবক্ষয়কে ঘিরেই মূলত তৈরি হয়েছে এই সংকট।ফলে প্রত্যাশা ও প্রাপ্তির যে দ্বদ্ব আমরা উপন্যাস জুড়ে বিভিন্ন মালো নরনারীর জীবনে লক্ষ করি; তা মালোদের গোষ্ঠীজীবনের ক্ষেত্রেও সত্য হয়ে ওঠে।অদ্বৈত মল্লবর্মণের 'তিতাস একটি নদীর নাম' উপন্যাসের ব্যক্তিজীবন বনান গোষ্ঠী-জীবনের এই প্রত্যাশা ও প্রাপ্তির দ্বন্দ্বের স্বরূপটিকেই পর্যায়ক্রনে তুলে ধরার চেষ্টা করব আলোচ্য প্রবন্ধে।

(১) প্রথনেই জীবনের এই প্রত্যাশা ও প্রাপ্তির দ্বন্দ কীভাবে প্রতিটি মালো নরনারীর ব্যক্তিগত জীবনে ধরা পড়েছে, তা উপন্যাস অবলম্বনে তুলে ধরা যেতে পারে----

এক্ষেত্রে প্রথনেই কিশোর আর অনন্তের মায়ের ট্র্যাজিক জীবনের কথায় আসা যাক। প্রত্যাশা ও প্রাপ্তির দ্বন্দ্ব এই দুই নরনারীর জীবনে যেভাবে লক্ষ করা যায়, তা এক ট্র্যাজিক সুরে পাঠক হৃদয়কে ভারাক্রান্ত করে তোলে। কিশোর মালোদের ছেলে, কিন্ত কিশোরের মনের গড়নটা আর পাঁচটা মালো পুরুষদের থেকে আলাদা। কিশোরের মধ্যে ঔপন্যাসিক দেখিয়েছেন এক রোমান্টিক সত্তাকে। নদীর সাথে তার সমম্পর্ক শুধুই জীবিকার নয়---বরং নদীপথে অজানার উদ্দেশ্যে পাড়ি দিতে তার রোমান্টিক সৌন্দর্যপিয়াসী মন সর্বদাই ব্যাকুল। আর এইভাবেই একদিন বন্ধু সুবলকে সঙ্গে নিয়ে মৎসশিকারের জন্য তিতাসের জলে নৌকা ভাসিয়ে সুদূরের উদ্দেশ্যে পাড়ি দেয় দেয় কিশোর। মাছ ধরা নয়, বরং নিত্য নতুনের আহবানেই যেন তার এই যাত্রা। আর এই যাত্রা পথেই শুকদেবপুরে বসন্ত উৎসবের দিন কিশোর খুঁজে পায় তার স্বপ্নের নায়িকাকে। যদিও এই নৎসশিকারের উদ্দেশ্যে রওনা হবার আগেই তাদের পাড়ার নেয়ে বাসন্তীর সাথে তার বিবাহ স্থির হয়েছিল, কিন্ত 
3 | তিতাস একটি নদীর নাম' : ব্যক্তিজীবন বনাম গোষ্ঠীজীবনের প্রত্যাশা ও প্রাপ্তির দ্বন্দ্ব

বাসন্তীকে বিবাহ করতে চায়নি কিশোর। কারণ কিশোরের মতে বিবাহ তাকেই করা যায়, যার সাথে কোনদিন কোন পরিচয় থাকেনা----নতুন করে মন তাকে চিনবে জানবে, তাতেই মনের সুখ।তাই যে বাসন্তীর সাথে তার নিত্যদিনের পরিচয়, তাকে বিবাহ করতে রাজি হয়না কিশোরের রোমান্টিক মন।বরং ভীন গাঁয়ের এই অপরিচিতার মধ্যেই কিশোর খুঁজে পায় তার মনের মানুষকে। তাকে নিয়েই ঘর বাঁধার স্বপ্ন দেখে কিশোর এবং ঈশ্বরের ইচ্ছায় তার সে প্রত্যাশা পূর্ণও হয়। কারণ ঐ গ্রামের মালোসমাজ কিশোরের সাথে নেয়েটির সম্পর্ককে সামাজিক স্বীকৃতি দেয়। কিন্ত বিধি যদি বাম হয়, তবে সুখ বুঝি কাছে এসেও দূরে সরে যায়! কিশোরের জীবনেও তাই ঘটেছে। নতুন বিবাহিতা স্ত্রীকে নিয়ে নতুন সাংসারিক জীবনের রঙিন স্বপ্ন দু চোখে ভরে কিশোর গ্রানের উদ্দেশ্যে রওনা হলেও সেই স্বপ্ন মাঝপথেই ভেঙে খান খান হয়ে গেছে। ডাকাতের দল মাঝরাতে কিশোরদের নৌকা থেকে টাকা পয়সার সাথে অপহরণ করে নিয়ে যায় তার সদ্যবিবাহিতা স্ত্রীকে।ফলে যে স্বপ্নকে মনে মনে লালন করেছিল কিশোর, এভাবে তাকে ভেঙে যেতে দেখে সেই আঘাত সহ্য করতে পারেনি কিশোরের কোমল মন, ফলে কিশোর হয়ে যায় সম্পূর্ণ উন্মাদ। এখানেই নিয়তির ছলনায় কিশোর হয়ে পড়ে এক ভাগ্যহত পুরুষ।

আর অন্যদিকে তার স্ত্রী অর্থাৎ অনন্তের মায়ের জীবনটাও যেন আগাগোড়া এলোনেলো। কিশোরের সাথে তার বিবাহটুকুই হয়েছিল, কিন্তু স্বানীর ঘরে এসে সুখে সংসার করা তার কপালে ছিল না। তাকে নিয়ে তার নিষ্ঠুর ভাগ্যের পরিহাস সত্যই বড় মর্মন্তদ! সেদিন ডাকাতের দ্বারা অপহৃত হয়েও শেষপর্যন্ত সে প্রাণে বেঁচে গিয়ে এক জেলে পরিবারে আশ্রয় পেয়েছিল। কিন্ত আমাদের মনে হয় তাকে নিয়ে তার নিষ্ঠুর ভাগ্যের যেন পরিহাস করার অনেক কিছুই বাকি ছিল সেদিন; তাই তো মৃত্যুর মুখোমুখি হয়েও সে প্রাণে বেঁচে যায়।কারণ এরপর তার ভাগ্যহত জীবনের যে পরিচয় আমরা পাই, তাতে তার অস্তিত্ব জুড়ে জেগে থেকেছে এক প্রবল প্রেম তৃষ্ণা----যার কোন নিবৃত্তি নেই।বস্তুত এই ঘটনার দীর্ঘ দিন বাদে কিশোরের ভালবাসার চিহ্ন স্বরূপ অনন্তকে সঙ্গে নিয়ে একদিন সে শ্বশুরের গ্রানে ফিরে আসে----প্রত্যাশা একটাই, হারিয়ে যাওয়া মনের মানুষের সন্ধান। আমরা জানি বিচ্ছেদের মধ্য দিয়েই প্রেম গাঢ়তা পায়, হারিয়ে ফিরে পাওয়ার মধ্যে মন এক নতুনত্বের স্বাদ পায়। কিন্ত হারিয়ে যাওয়া মানুষটা কিংবা যার জন্য অপেক্ষা করে থাকে মন, সেই মানুষটাই যদি বদলে যায়----তবে আর তা বুঝি হারানো প্রাপ্তির আনন্দ বহন করে না। অনন্তের মায়ের ক্ষেত্রেও তাই ঘটেছে। যে মনের মানুষের সন্ধানে দীর্ঘ দিন পর অনন্তকে সঙ্গে করে আবার সে স্বামীর গ্রানে ফিরে এসেছিল, তার সেই প্রত্যাশা স্বপ্ন ভঙ্গের যন্ত্রণা হয়েই ফিরে এসেছে যখন সে জানতে পারে ঐ গ্রানের পাগল কিশোরই তার সেই হারিয়ে যাওয়া স্বামী। তবু অন্তরের ভালবাসা আশা ছাড়ে না, বরং পাগল স্বামীকে সুস্থ করে তোলার সংকল্প নেয় সে মনে মনে। তাই তো সুবলের বউয়ের সাথে কথোপকথন কালে রসিকতার ঢঙে বলা এই কথাটি কিন্ত অনন্তের মায়ের মনের কথাই ছিল----

"তুমি তো তার মনের মানুষ মিলাইয়া দিতে পার না।

তা পারি না! তবে চেষ্টা কইরা দেখতে পারি, আমি নিজে তার মনের মানুষ হইতে পারি কিনা।"২

কিন্ত কিশোরকে মনের মানুষ করে জীবনে পাওয়া আর হয়ে ওঠে না এই দুর্ভাগিনী নারীর--বরং চিরতরে হারাতেই হয়েছে। কারণ দোল উৎসবের দিন কিশোরকে রঙ মাখাতে গেলে পাগলামির ঝোঁকেই হোক কিংবা পূর্ব স্মৃতির বিকারবশত কিশোর অনন্তের মায়ের ওপর শারীরিক নির্যাতন করে বলে। আর এই অবস্থায় পাগল 
কিশোরের হাত থেকে অনন্তর মাকে বাঁচাতে পাড়াপড়শীরা কিশোরের ওপর অমানুষিক প্রহার চালায়। যে অত্যাচার সহ্য করতে না পেরে সে মারা যায়। অনন্তের মায়ের সব থেকে বড় যন্ত্রণা এখানেই হয়তো যে, পাগল স্বামীকে সুস্থ করে তুলতে গিয়ে সে নিজেই যেন প্রকারান্তরে তার মৃত্যুর কারণ হয়ে দাঁড়িয়েছে। ফলে এই আঘাত সহ্য করা সম্ভব হয়নি অনন্তের মায়ের পক্ষে, তাই কিশোরের মৃত্যুর দুদিন যেতে না যেতে সে নিজেও মারা যায়।শুকদেবপুরের এক বসন্ত উৎসবের দিন যে প্রেনের সূচনা হয়েছিল, আর এক বসন্ত উৎসবের দিনে এভাবেই যেন তার পরিসমাপ্তি। মানবজীবনে নিয়তির প্রভাব যে কতখানি নিষ্ঠুর হতে পারে, এই দুই ভাগ্যহত নরনারীর জীবনই তার সাক্ষ্য বহন করে। জীবন তাদের যেন কিছুই দিতে পারেনি, বরং তাদের অন্তরে জাগিয়ে রেখেছে শুধুই প্রত্যাশা আর প্রাপ্তির দ্বন্দ্ব। এক প্রবল প্রেম তৃষ্ণা নিয়েই যেন এই দুই নায়ক নায়িকা জীবন রঙ্গভূমি থেকে চিরবিদায় নিয়েছে। বিশেষত অনন্তের মায়ের করুণ জীবিনকথা পাঠক হৃদয়কে বেশি করে ছুঁরে যায়। কারণ কিশোর তো তার প্রত্যাশার অপমৃত্যুর সাথে সাথে পাগল হয়ে চেতনা হারিয়েছে, কিন্ত অনন্তর মাকে বেঁচে থেকে নিত্য জীবনের অপূর্ণতার যন্ত্রণাকে বহন করে যেতে হয়েছে। তার জীবনের কোন প্রত্যাশাই পূর্ণতা পায়নি, এমনকি লক্ষ করার সে এই উপন্যসের অনাম্নী অঙ্গনা----ঔপন্যাসিক তার কোন নাম পর্যন্ত দেননি। আর এপ্রসঙ্গে মনোজ্ঞ একটি বক্তব্য তুলে ধরেছেন প্রাবন্ধিক ড. রুনেলা বন্দ্যোপাধ্যায়-----

"যার কোন মর্যাদা নেই, যার প্রেম প্রতিষ্ঠা পায় না, যে সন্তানের পরিচয় দিতে পারে না, যে স্বামীকে উন্মার্গগামিতা থেকে স্বাভাবিকতায় আনতে চেয়েও পারে না সমাজের প্রতিকূলতায়, কী প্রয়োজন তার নানে ? 'নাম' তো নামবাচক শুক্ষ একটা শব্দ নয়, যা উচ্চারণ করে কারো সাড়া নেওয়া যায়, সে অনেক অনেক কিছু। নানের সঙ্গে জড়িয়ে থাকে একটা জীবনের ইতিহাস, তার উত্থান, তার ভালবাসা, তার ব্যক্তিত্বের ঠিকানা, তার স্থিতি, তার স্বাভাবিক পরিণতি। কী আছে অনন্তর মায়ের?"৩

সমালোচকের এই বক্তব্য থেকেও আমরা অনন্তর মায়ের অপূর্ণ জীবনের স্বরূপটিকে বুঝে নিতে পারি। কিশোর আর অনন্তর মায়ের জীবনের এই অপূর্ণতার আখ্যান, তাদের ভাগ্যহত করুণ জীবনকথা তাই শুধু পাঠক হৃদয়কে না , যেন ভারাক্রান্ত করে তোলে তিতাসের আকাশ বাতাসকেও।

আবার কিশোরের জীবনের এই অপ্রত্যাশিত বিপর্যয়কে ঘিরে প্রত্যাশা প্রাপ্তির আরেক দ্বন্ব এই উপন্যালে লক্ষ করা যায় কিশোরের বৃদ্ধ পিতা-মাতার জীবনে।আমরা জানি, সন্তানের সুখেই পিতা মাতার সুখ। তাই তো সেই মধ্যযুগের বাংলা সাহিত্য 'অন্নদামঙ্গল' কাব্যেও দেবী অন্নপূর্ণার উদ্দেশ্যে ঈশ্বরী পাটনীর চিরন্তন প্রার্থনা-'আমার সন্তান যেন থাকে দুধে ভাতে'। সন্তানকে মানুষ করে তোলা যেমন পিতা মাতার দায়িত্ব, তেমনি উপযুক্ত বয়সে সন্তানের বিবাহ দেওয়া, বিশেষত পুত্র সন্তানের ক্ষেত্রে তার বিবাহ দিয়ে তার সুখী সংসারের ছবিটা দেখে যাবার ইচ্ছা প্রত্যেক পিতা মাতারই মনের সুপ্ত বাসনাস্বরূপ। কিশোরের পিতা মাতাও তাদের কিশোরের বিবাহ দিতে চেয়েছিল বাসন্তীর সাথে। মাছ ধরতে যাওয়া কিশোর বাড়ি ফিরে এলেই বাসন্তীর সাথে তার বিবাহ হবে, এমনটাই যখন স্থির হয়ে আছে-----ঠিক তার মাঝেই ঘটে গেল এক বিপর্যয়।কিশোর বাড়ি ফিরে এল সম্পূর্ণ পাগল অবস্থায়।ফলে কিশোরের বিবাহকে ঘিরে তার পিতা মাতার সমস্ত প্রত্যাশা, সমস্ত স্বপ্ন যেন ভেঙে খান খান হয়ে যায় এই ঘটনায়। দুদিন পরে উৎসবের আনন্দে যে পরিবারের মেতে ওঠার কথা, এই আকস্মিক দুর্ঘটনা যেন সেই পরিবারটিকে একেবারে ছন্নছাড়া করে দেয়।শুধু তাই নয়, যে বাসন্তীর তাদের ঘরের বউ হয়ে আসার কথা, এক রাতে যেন তাদের চোখের সামনেই তার বিবাহ হয়ে গেল কিশোরেরই বন্ধু সুবলের 
5 | তিতাস একটি নদীর নাম' : ব্যক্তিজীবন বনাম গোষ্ঠীজীবনের প্রত্যাশা ও প্রাপ্তির দ্বন্দ্ব

সাথে।নিয়তির নিষ্ঠুর পরিহাস ছাড়া একে আর কিছুই বলা চলে না। এ যেন নিজেদের স্বপ্নের অপমৃত্যুকেই আরো একবার শুধু অসহায়ের মতো চেয়ে চেয়ে দেখা। তাই লেখক যখন বলেছেন----

"একদিন সুবলার সঙ্গে বাসন্তীর বিবাহ হইয়া গেল। সেই এক রাত। আকাশে চাঁদ আছে তারা আছে। দীনবনাথের উঠানে কলাগাছের তলায় বাসন্তীকে সুবল হাতে হাত দিয়ে বউ করিতেছে। মেয়েরা গীত গাহিতেছে হুলুধ্বনি দিতেছে। জোরে জোরে বাজনা বাজিতেছে।এত জোরে বাজিতেছে যেন রামকেশবের কানের পর্দা ছিঁড়িয়া যাইবে। একটা টিমটিনে আলোর সামনে রামকেশব তামাক টানিতেছে। হুকার শব্দে বাজনার শব্দ ঢাকিবার চেষ্টা করিতেছে। তার পাশে বুড়ি বসিয়া ঝিমাইতেছে।......... অনেক রাত অবধি সেই বাজনা চলিল। তারপর এক সময় উহাও নিস্তব্ধ হইয়া গেল।তখন বুঝি বিবাহবাড়ির সকলেই ঘুমাইয়া পড়িয়াছে। কিন্ত বুড়া বুড়ির চোখে সে রাতে আর ঘুম আসিল না।"8

তখন এই অংশে স্বপ্ন ভঙ্গের যন্ত্রণায় দুই অসহায় পিতা মাতার দীর্ঘশ্বাস আর অব্যক্ত হাহাকার যেন ফুটে ওঠে। বিশেষত নিজেদের জীবৎদশাতেই সন্তানের মৃত্যুর থেকে মর্মান্তিক ঘটনা কোন পিতা মাতার জীবনেই আর কিছু হতে পারে না। আর নিয়তির লিখনে কিশোরের পিতা মাতাকে সেই নিষ্ঠুর অভিজ্ঞতারও সাক্ষী থাকতে হয়েছে।বস্তুত জীবনের প্রত্যাশা-প্রাপ্তির চিরকালীন দ্বন্দ্বটিকেই যেন অদ্বৈত মল্লবর্মণ তুলে ধরেছেন এই অংশে সন্তানের জীবনের বিপর্যকে ঘিরে তার পিতা মাতার জীবনের অসহায়তা ও যন্ত্রণার আবহে।

এই উপন্যালে অপূর্ণতার আরেক আখ্যান গড়ে উঠেছে বাসন্তী ও সুবলের ভাগ্যহত জীবনকে ঘিরে। কিশোর ও সুবলের সাথে একসঙ্গেই বড় হয়েছে বাসন্তী। এই দুই মালো তরুণই তাদের স্বতঃস্ফূর্ত প্রাণপ্রাচুর্যে ভরিয়ে রেখেছিল এই মালো তরুণীর মন। যদিও বাসন্তীর মনের দুর্বলতা কিছুটা কিশোরের প্রতি। এই অবস্থায় কিশোরের সঙ্গে বাসন্তীর বিবাহ স্থির হয় দুই পরিবারের পক্ষ থেকে। কিন্ত কিশোরের সাথে ঘর বাঁধা বাসন্তীর আর হয়ে ওঠে না। কারণ বিবাহের আগে তিতাসের বুকে নৌকা ভাসিয়ে মাছ ধরতে যাওয়া কিশোর নতুন করে এক নারীর প্রেনে পড়ে এবং তাকে বিবাহও করে। যদিও বাড়ি ফেরার পথে তাকে হারিয়ে পাগল অবস্থায় ফিরে আলে কিশোর, কিন্তু স্বাভাবিক অবস্থায় ফিরে এলেও আর বাসন্তীকে ঘরে নেওয়া বিবাহিত কিশোরের পক্ষে সম্ভব হত না। বাসন্তীর জীবনে প্রথম স্বপ্ন ভঙ্গের যন্ত্রণা এখানেই। কিন্ত নিষ্ঠুর ভাগ্যের পরিহাস তার জীবনে যেন এখানেই শেষ হয়ে যায় না। নতুন করে সুবলের সাথে বাসন্তীর বিবাহ স্থির হয়, কিন্ত এই বিবাহও তাকে কাঙ্খিত সুখ এনে দিতে পারেনি। কারণ বিবাহের মাত্র কদিনের মধ্যেই মাছ ধরতে গিয়ে প্রাণ হারায় সুবল।ফলে শাঁখা সিঁদুর খুইয়ে বাসন্তীকে হতে হয় বিধবা। এপ্রসঙ্গে দু- এক কথায় সুবলের ভাগ্যহত জীবনের কথা বলা আবশ্যক। কিশোরের সাথে বাসন্তীর বিবাহ স্থির হলেও সুবল বাসন্তীকে মনে মনে ভাল বাসত। তাই যে মুহূর্ত্ত সে কিশোরের কাছে জানতে পারে কিশোর বাসন্তীকে বিবাহ করতে চায় না, সেই মুহূর্ত থেকে সুবলের মনে আশার সঞ্চার ঘটে। তাই উৎসাহের সাথে সে কিশোরের কাছে জানতে চায়----

"সকল কথা শুনিয়া সুবল আনন্দে লাফাইয়া উঠিয়া বলে, 'দাদা। তা হইলে বাসন্তীরে তুমি এখানেই পাইয়া গেলা। অখন দেশের বাসন্তীরে কার হাতে তুইল্যা দিবা কও।'

'তোর হাতে দিয়া দিলাম।'

সুবলের মনে একটা আশার রেশ গুন গুন করিয়া উঠে।"৫ 
বাসন্তীকে নিয়ে নতুন করে ঘর বাঁধার স্বপ্ন দেখে সুবল। ঈশ্বরের ইচ্ছায় তার সেই স্বপ্ন পূর্ণও হয়। কিন্ত তার নিষ্ঠুর নিয়তি যেন তাকে সুখের সুধাভান্ডটুকু ক্ষণিকের জন্য দেখার সুযোগটুকুই করে দিয়েছে, তা পরখ করা আর তার ভাগ্যে ঘটে না। তাই তো নদীতে মাছ ধরতে গিয়ে প্রবল ঝড়ের মাঝে নদীর চরে নেনে নৌকাকে সামাল দিতে গিয়ে সে নিজে নৌকা চাপা পড়ে নৃশংস ভাবে প্রাণ হারায়।নিয়তির নিষ্ঠুর ছলনায় মানবভাগ্যের নিদারুণ পরিণতিরই সাক্ষ্য বহন করে সুবলের জীবনকথা। আর বাসন্তীর মতো অভাগিনী নারী বুঝি বাংলা সাহিত্যে খুব কমই আছে। কুমারী বয়সে বাসন্তী মাঘমন্ডল ব্রত করেছে। আমরা জানি প্রত্যেক ব্রতের পেছনেই নারী মনের কোন না কোন কামনা কাজ করে। আর মাঘমন্ডল ব্রতের ক্ষেত্রে মূলত কুমারী নারীমনের সুখী দাম্পত্যজীবনের কামনাই প্রতিফলিত হয়। কিন্ত কুমারী বয়সের সেই ব্রত পালন তার জীবনে কাঙ্খিত সুখ এনে দিতে পারেনি। দু- দুজন পুরুষ বাসন্তীর জীবনে এসেছে, কিন্ত কেউই বাসন্তীকে সুখী করতে পারেনি। মরীচিকা যেমন তৃষ্ণার্ত পথিককে ছলনা করে, বাসন্তীর জীবনেও সুখ তেমনি তাকে নিয়ে শুধুই ছলনা করেছে, তার তৃষ্ণা বাড়িয়ে দিয়েছে,-------কিন্ত নিবৃত্তি ঘটায়নি।আর এই তিক্ত জীবন অভিজ্ঞতা বাসন্তীর পুরুষ সমাজের প্রতি বিশ্বাসকেই নষ্ট করে দিয়েছে।তাই তো পাগল কিশোরের প্রতি অনন্তের মাকে মুগ্ধ হতে দেখে পুরুষমানুষ সম্পর্কে সে তার মনের নিদারুণ অভিজ্ঞতার কথা জানিয়েছে---

" পুরুষ মানুষ দিয়া কি হইব। তারা বৃষ্টির পানি-ফোঁটা, ঝরলেই শেষ। তারা জোয়ারের জল। তিলেক মাত্র সুখ দিয়া নদীর বুক শুইষ্যা নেয়।" ৬

সুবলকে বিবাহ করে কোন সুখ বাসন্তীর ভাগ্যে ঘটেনি----বরং যা জুটেছে, তা হলো 'সুবলের বউ' এই নামটুকু। তাই আমাদের মনে হয় ঔপন্যাসিক যেন বার বার এই ' সুবলের বউ' নামটিকে ব্যবহার করে তার বিড়ম্বিত জীবনের পরিচয়টিকেই আমাদের স্মরণ করিয়ে দিতে চেয়েছেন। আবার এই 'সুবলার বউ' নামকরণের মধ্যে বাসন্তীর জীবনের যে অপূর্ণতার প্রতি আমাদের বিশেষভাবে দৃষ্টি আকর্ষণ করাতে চেয়েছেন অধ্যাপক অচিন্ত্য বিশ্বাস, তা এপ্রসঙ্গে তুলে ধরতে পারি----

"প্রেনের যে বারিধারায় বাসন্তী স্নাত হতে চেয়েছিল, কিশোরের অন্যত্র বিবাহ ও উন্মাদনায় সেই ধারা স্নান থেকে বাসন্তী বঞ্চিত হয়। সে তৃষ্ণার্ত থেকে যায়। সুবলার বউ নামকরণের মধ্যে বাসন্তীর অতৃপ্ত তৃষ্ণাটিই পাঠকের মনে জেগে থাকে।"৭

আসলে কিশোরের প্রতিই যে বাসন্তীর মনের দুর্বলতা ছিল, তা আমরা আগেই বলেছি। এই কিশোরের সাথেই ঘর বাঁধতে চেয়েছিল বাসন্তী, 'কিশোরের বউ' এই নামটাই হয়তো ছিল তার কাঙ্খিত; কিন্ত ভাগ্যের পরিহাসে সে হয়ে গেল 'সুবলের বউ'। যে সুবলও আজ তার জীবনে নেই; অথচ নিয়তির পরিহালে তার নামটুকু চিরকালের জন্য তার জীবনের সাথে অচ্ছেদ্য ভাবে জড়িয়ে গেছে। হারিয়ে গেছে তার মা বাবার দেওয়া বাসন্তী নাম। এইভাবে জীবনের চিরকালীন প্রত্যাশা ও প্রাপ্তির দ্বন্দ্বই যেন বড় হয়ে উঠতে দেখা বাসন্তীর জীবনে। আর তেমনি সুবল এই উপন্যাসের আরেক হতভাগ্য পুরুষ, সুখ যার চির অধরাই থেকে গেছে।

অন্যদিকে এই উপন্যাসে বাসন্তীর জীবনেই প্রত্যাশা ও প্রাপ্তির আরেক দ্বন্দ্ব, আরেক দোলাচলতা ধরা পড়েছে তার মাতৃহৃদয়ের আশা আকাঙ্খাকে ঘিরে।প্রত্যেক নারীই যেমন একটা সুন্দর সংসারের স্বপ্ন দেখে, তেমনি সে তার নারী জীবনের সার্থকতা খুঁজে পেতে চায় আপন সন্তানের মধ্যে। বিশেষত একজন বিধবার নিঃসঙ্গ নারী জীবনে বাঁচার একমাত্র অবলম্বনই তার সন্তান। সে দিক থেকে কিশোরের স্ত্রী তথা অনন্তের মায়ের জীবনে 
7 | তিতাস একটি নদীর নাম' : ব্যক্তিজীবন বনাম গোষ্ঠীজীবনের প্রত্যাশা ও প্রাপ্তির দ্বন্দ্ব

একটা সান্ত্বনা ছিল। কিন্ত সুবলের সঙ্গে স্বল্প দিনের বিবাহিত জীবন বাসন্তীকে তার মাতৃহৃদয়ের সেই আকাঙ্খা চরিতার্থতা করার সুযোগ করে দিতে পারেনি। এই অবস্থায় অনন্তকে ঘিরেই সুবলের বউ তথা বাসন্তীর সুপ্ত জননীসত্তা যেন প্রকাশের পথ খুঁজে পায়। তাই অনন্তের যাতে কষ্ট না হয়, তার জন্য নিজের মা বাবার সাথে দিনরাত অশান্তি করেও সে অনন্ত ও তার মাকে সাহায্য করেছে। মূলত অনন্তকে ঘিরেই সুবলের বউ আর অনন্তের মা'র মধ্যে সখীত্বের সম্পর্ক গড়ে ওঠে।এমনকি আমরা দেখি, অনন্তের মায়ের মৃত্যু হলে, এই সুবলের বউই নিজে দাঁড়িয়ে থেকে মায়ের শ্রাদ্ধাদির কাজ অনন্তকে দিয়ে করিয়েছে। আসলে অনন্ত সুবলের বউকে মাসী বলে ডাকলেও সে অনন্তের দ্বিতীয় মা স্বরূপ এবং সেই মায়ের দায়িত্বই সে চিরকাল পালন করেছে। কিন্ত শেষ পর্যন্ত নিজের কাছে অনন্তকে ধরে রাখতে পারেনি সে----- আর এখানেই তার মাতৃহৃদয়ের যন্ত্রণা। কারণ সুবলের মৃত্যুর পর বিধবা বাসন্তীকে আবার সেই আগের মতোই মা বাবার সংসারেই ফিরে আসতে হয়েছে।তাই নিত্যদিন মা বাবার সঙ্গে ঝগড়াঝাঁটি করে সন্তানসম অনন্তকে নিজের কাছে রাখা তার পক্ষে সম্ভব হয় না। এক্ষেত্রে সুবলের বউ পরিস্থিতির শিকার, তাই বাধ্য হয়েই একদিন সে অনন্তকে গাল মন্দ করে তাড়িয়ে দেয়। কিন্ত অনন্ত সেদিন তার এই মাসীর শুধু মুখের কথাটুকুই শুনেছিল, ভেতরের মাতৃহৃদয়ের যন্ত্রণাটা দেখতে পায়নি ---- তবে পাঠকের পক্ষে তা বুঝে নেওয়া মুশকিল হয়নি। আর তাই তো দীর্ঘ দিন পরে নৌকার বাইচ খেলার দিনে অনন্তকে আবার খুঁজে পেয়ে তাকে বুকে জড়িয়ে ধরার জন্য ব্যাকুল হয়ে ওঠা বাসন্তীর মাতৃহৃদয়ের পরিচয়টুকু ঔপন্যাসিক আমাদের কাছে যথার্থই তুলে ধরেছেন----

"মাসী ডাকে আকৃষ্ট হইয়া সুবলার বউ চকিতে ঘাড় ফিরাইয়া দেখিল, তারপর সে উদ্দান হইয়া বলিয়া উঠিল , অনন্ত? আমার অনন্ত!

দুই নৌকার বাতা লাগানো ছিল। লাফাইয়া সে এ নৌকাতে আসিয়া উঠিল এবং অনন্তর দিকে দুই হাত বাড়াইয়া দিল। মাসী মাসী বলিয়া অনন্তও হাত বাড়াইল। দেখিল, মাসীর দুই চোখে অশ্রুর বন্যা বহিয়াছে।"b-

দীর্ঘ সময় পর দূরে থাকা সন্তানকে কাছে পেলে যেমন একজন মায়ের আহ্লাদের শেষ থাকে না, অনন্তকে ফিরে পেয়ে সুবলের বউয়ের মনের অবস্থাও তাই। শুধুমাত্র পরিস্থিতির শিকার হয়েই যে একদিন সে শত যন্ত্রণা সত্বেও তা গোপন করে অনন্তকে দূরে সরিয়ে দিয়েছিল, আজকের এই দৃশ্যই যেন সে সাক্ষ্য বহন করে। কিন্ত এরপরই যে তার মাতৃসত্তাকে এক কঠিন কঠোর বাস্তবের মুখোমুখি দাঁড়াতে হবে, তা যেন ভাবতেই পারেনি সুবলের বউ। বর্তমান আশ্রয়দাত্রী উদয়তারার প্ররোচনায় অনন্ত পূর্বের কথা স্মরণ করে(অনন্তকে সুবলের বউয়ের তাড়িয়ে দেবার ঘটনা) নিষ্টুর ব্যবহারে ফিরিয়ে দিয়েছে তার প্রতি সুবলের বউ তথা তার মাসীর স্নেহ ভালবাসার সমস্ত আবদার ও আবেদনকে। ফলে অনন্তকে ঘিরে সুবলের মাতৃসত্তা যেন এক মূহৃর্তে তার দাঁড়ানোর ভিত্তুভূমিটিকে হারিয়ে এক প্রবল অস্তিত্ত্বের সংকটের মুখোমুখি হয়েছে-----

"তুইও কি আমার পর হইয়া গেলি অনন্ত।"

--আপন তো কোন কালে নই মাসী। মার সই তুমি। মা যতদিন ছিল, তোমার কাছে আমার আদরও ততদিনই ছিল। মা মরিয়া গেল, সে আদরো একদিন হাট- বাজারের মতই ভাঙ্গীয়া পড়িল।

ভাঙ্গিয়া পড়িল! কি করিয়া তুই বুঝিলি যে, ভাঙ্গিয়া পড়িল?"৯ 
যে অনন্তকে নিজের ছেলের মতো ভালবেসেছে এই সন্তানহীনা রমণী, যার জন্য একদিন নিজের মা বাবার সঙ্গে পর্যন্ত বিবাদ করতেও ছাড়েনি; সেই অনন্তের এই হৃদয়বিদারক, বেইমানের মতো কথা- বার্তায় আজ আর নিজেকে স্থির রাখতে পারেনি সুবলের বউ।তার মাতৃসত্তা অনন্তের এই নিষ্ঠুর ব্যবহারে প্রতিহত হয়ে মারমুখী হয়ে ওঠে অনন্তের প্রতি। কিন্ত অনন্তের উপর যে অধিকার তার একদিন ছিল, সেই অধিকার যে আর আজ নেই তার, একথা যেন বুজে উঠতে পারেনি সুবলের বউ। তাই অনন্তের গায়ে হাত তোলার পাল্টা জবাব হিলেবে উদয়তারার দলবল ঝাঁপিয়ে পড়ে তার ওপর। কেউই বুঝতে চায়লনা সুবলের বউয়ের মাতৃহৃদয়ের যন্ত্রণার দিকটি-----না উদয়তারা, আর না অনন্ত নিজে। উদয়তারার দলবলের অমানুষিক প্রহারে সেদিন দেহ ও মনে একরাশ যন্ত্রণার বোঝা নিয়ে কোনক্রনে বাড়ি ফিরে এসেছিল সে। অনন্তের বউ সম্পর্কে ঔপন্যাসিক বলেছেন-

"ছেলেবেলায় মা বাবা তাকে অত্যন্ত ভালবাসিত। কোনদিন তারা তার গায়ে হাত তোলে নাই। মালোদের পাড়ার নেয়েতে নেয়েতে ঝগড়া হয়, গালাগালি করে। কিন্তু পাড়ার পাঁচজনের কেউ কোনদিন তার প্রতি কটুবাক্য প্রয়োগ করে নাই। পাড়ার মধ্যেতার নিজের একটা মর্যাদা ছিল।"১০

কিন্তু আজ আর সেই মর্যাদা যেন সে ধরে রাখতে পারলনা, কারণ যেভাবে উদয়তারার দলবলের কাছে সর্বসমক্ষে সে লাঞ্ছিত হয়েছে, তাতে লজ্জা আর অপমানে কারোর কাছে মুখ দেখানোর অবস্থা থাকে না তার।শুধুমাত্র সন্তানস্নেহে অনন্তকে ভালবেসে যে এতখানি দাম তাকে দিতে হবে , তা যেন স্বপ্নেও কোন দিন ভাবতে পারেনি এই সন্তানহীনা রমণী। দেহে মনে প্রচন্ড যন্ত্রণা নিয়ে সেদিন রাতে যখন নিজের মাকে জড়িয়ে সুবলের বউ এক পরম শান্তিতে মনে মনে ভেবেছে---

"শরীরের ব্যথায় মাঝে মাঝে ঘুম ভাঙ্গে, আবার ঘুম আলে। এই চেতন অবচেতনের ফাঁকে ফাঁকে কেবলই তার মনে হইতে থাকে সংসারে কেবল মা-ই সত্য আর কিছু না"।১১

-- তখন এই কথার মধ্যে একদিকে যেমন তার এই দুঃসময়ে নিজের মায়ের ওপর একান্ত নির্ভরতার দিকটি ধরা পড়ে, তেমনি তার মাতৃসত্তার অভিমানী কণ্ঠস্বরটিও এখানে স্পষ্ট। আসলে এই পৃথিবীতে মা-ই একমাত্র সত্য; আর তার জন্য সন্তানকে গর্ভে ধারণ করতে হয়। অনন্তকে সুবলের বউ নিজের গর্ভে ধারণ করেনি। তাই তো অনন্তকে নিজের সন্তানের মতো ভালবেসে, তার জন্য শত বিপত্তি মাথায় নিয়েও সে শুধুই তার মাসী হয়েই থেকে গেছে----মা হয়ে উঠতে পারেনি। যদি পারত, তবে আজ উদয়তারার কথায় অনন্ত তার মাতৃসত্তার নিঃস্বার্থ আবেদনকে এভাবে রূঢ়তার সাথে ফিরিয়ে দিতে পারত না। সুবলের বউয়ের নাতৃহৃদয়ের এই অভিনানী সুরটিই এখানে যেন ধরা পড়ে। আর এইভাবে সমগ্র উপন্যাস জুড়ে অনন্তকে ঘিরে সুবলের বউয়ের মাতৃহৃদয়ে জেগে থেকেছে এক চির অতৃপ্তি, প্রত্যাশা-প্রাপ্তির নিরন্তর দ্বন্দ্ব-----যার কোন নিবৃত্তি নেই।তাই তো মৃত্যুর ঠিক আগের মুহূর্তে যখন অনন্তের বিবাহের ছবি স্বপ্নের মতোই ভেলে এসেছে তার মনোজগতে, তখন তার চিন্তার বিষয় হয়েছে--অনন্তের বিবাহের দিন কে তার মায়ের আসন নেবে।আর এক্ষেত্রেও সেই একই দোলাচলতা তথা অনিশ্যতাতা জাত দ্বন্দ্ব ধরা পড়েছে, কারণ প্রথনে তার নিজের কথা মনে এলেও পরক্ষণেই অনন্তের মায়ের দাবি নিয়ে ভেলে উঠেছে উদয়তারা, রমুর মায়ের মুখ।

অন্যদিকে প্রত্যাশা প্রাপ্তির আরেক দ্বন্দ্ব এই উপন্যাসে আমরা লক্ষ করি অনন্তবালার চিরবঞ্চিত উপেক্ষিত জীবনে। অনন্তকে ভালবেসে সারাজীবন অপেক্ষা করে থেকেছে এই নারী, কিন্তু তার সেই প্রত্যাশা কোনদিনই 
9 | তিতাস একটি নদীর নাম' : ব্যক্তিজীবন বনাম গোষ্ঠীজীবনের প্রত্যাশা ও প্রাপ্তির দ্বন্দ্ব

পূর্ণতা পায়নি। ছোটবেলায় অনন্তের সাথে তার এক নিষ্পাপ প্রেনের সম্পর্ক গড়ে ওঠে, যদিও প্রেনের স্বরূপ উপলপধ্ধির মতো মনের গড়ন তাদের দুজনেরি হয়তো ছিলনা সেদিন। তাদের নানের মধ্যেও এক অদ্ভুত মিল--যা তাদের দুজনের জীবন সম্পর্কে সৃষ্টিকর্তার কোন শুভ ইঙ্গিত বলেই মনে হয় পাঠকের কাছে। অনন্তবালার পরিবার থেকে অন্তকে জামাই করার কথাও ওঠে, যা অনন্তবালার কথা থেকেই জানা যায়।কিন্ত তাদের দুজনের এই নিষ্পাপ সম্পর্ক, যার একটা সুন্দর পরিণতির প্রত্যাশা পাঠক হৃদয়ে তৈরি হয়েছিল শুরুর দিকে------তা কোন পরিণতি পায়নি। বরং এক পক্ষের(অনন্তবালার) প্রত্যাশা নিয়ে পথ চেয়ে থাকার মধ্যেই যেন তার পরিসমাপ্তি। অনন্ত পড়াশুনার সূত্রে শহরে চলে যায়। যাবার আগে সে অনন্তবালাকে কথা দিয়ে যায় যে, একদিন সে আবার তার কাছে ফিরবে আর সেদিন অনন্তবালা যা বলবে, অনন্ত তাই শুনবে।অনন্তের দেওয়া এই কথাকেই সম্বল করে সারা জীবন তার জন্য পথ চেয়ে থেকেছে অনন্তবালা। অথচ অনন্ত তার দেওয়া এই কথা রাখেনি। আমরা দেখেছি অনন্তের অপেক্ষায় মালোসমাজে কতখানি আত্মগ্লানি ও লাঞ্হনা নিয়ে দিন কাটাতে হয়েছে অনন্তবালাকে।লেখকের কথায়-----

"দিনদিনই তাকে একটু একটু করিয়া বড় দেখায়। শেষে মা খুড়িনাদের চোখেও দৃষ্টিকটু হইয়া পড়িল। তার চাইতে ছোট মেয়েরা দেখিয়া মাঝে মাঝে ছড়া কাটে, 'অনন্তবালা ঘরের পালা, তারে নিয়ে বিষম জ্বালা।"১২

মালো সমাজে তার বয়সী মেয়েরা যখন একে একে স্বামীর ঘরে চলে যাছে, তখন গ্রাম সমাজের শত লাঞ্হনা আর অপমান মুখ বুজে সহ্য করেও বিবাহের জন্য আসা সব সম্বন্ধকে ফিরিয়ে দিয়েছে সে। কারণ অনন্তকে ভুলে আর কারো সাথে ঘরবাঁধা তার পক্ষে সম্ভব নয়।অথচ যার জন্য তার এই প্রতীক্ষা, শহরে গিয়ে বাবু বেনে যাওয়া সেই অনন্তের আজ মনেই পড়েনা তার সেই ছোটবেলার সাথী অনন্তবালাকে। তাই তো বনমালীর হাত দিয়ে উদয়তারা ও সুবলের বই তথা তার মাসীর জন্য কাপড় কিনে পাঠিয়ে দিলেও অনন্ত বালার কথা আজ একবারের জন্য মনেও পড়েনি অনন্তর। আমরা দেখেছি, কতখানি আগ্রহ আর প্রত্যাশা নিয়ে অনন্তবালা বনমালী দাদার কাছে জানতে চেয়েছে, শহর থেকে তার অনন্ত তার জন্যও কাপড় কিনে পাঠিয়েছে কিনা।কিন্ত বনমালী দাদার কাছ থেকে যে কঠিন সত্য সে শুনেছে তাতে অনন্তকে ঘিরে তার সব স্বপ্ন ভেঙে গেছে, অনন্তর জন্য তার এই পথ চেয়ে থাকার অর্থহীনতাই স্পষ্ট হয়ে উঠেছে। অনন্তবালার জীবনের প্রত্যাশা আর প্রাপ্তির দ্বন্দ্ব এখানেই।মানুষ আশা করে থাকে এক, কিন্ত বাস্তবে হয় যেন আর এক। যে যার জন্য অপেক্ষা করে , সে তার কথা মনে রাখেনা। মানবজীবনের সেই চিরন্তন প্রত্যাশা আর প্রাপ্তির বিরোধই যেন সত্য হয়েছে অনন্তবালার জীবনে।

(২)এবার মালোদের গোষ্ঠীজীবনের ক্ষেত্রে এই প্রত্যাশা ও প্রাপ্তির দ্বন্দ্ব কিভাবে ধরা পড়েছে, সেই প্রসঙ্গে আলোকপাত করা যাক----

বস্তুত 'তিতাস একটি নদীর নাম' যেমন একটি নদী কেন্দ্রিক উপন্যাস, তেমনি একটি আঞ্চলিক উপন্যাসও বটে। আর আঞ্চলিক উপন্যাসের অন্যতন লক্ষণ হলো--local colour কে ফুটিয়ে তোলা। একটি জনগোষ্ঠীর নিজস্ব সংস্কৃতিকে তুলে ধরে এই উপন্যাস, যার সাথে ঐ জনগোষ্ঠীর থাকে নাড়ীর যোগ----তাদের ঐক্যবদ্ধ জীবনের বন্ধন তাদের নিজস্ব সংস্কৃতি। আর সেই সংস্কৃতিকে নতুন কালের করাল গ্রাস থেকে রক্ষা করাই তাদের জীবনের ব্রত স্বরূপ। কিন্তু কালের স্রোতকে ঠেকিয়ে রাখা কারো পক্ষেই সম্ভব নয়। আর তাই প্রত্যাশা প্রাপ্তির দ্বন্দ্ব আরেক মাত্রা পেয়েছে এই উপন্যাসে কালের স্রোতে মালোদের নিজস্ব সংস্কৃতির সংকটকে ঘিরে। 
এপ্রসঙ্গে আমাদের মনে পড়ে যায় তারাশঙ্করের 'হাঁসুলী বাঁকের উপকথা' উপন্যাসটির কথা। বনোয়ারী কাহারদের নিজস্ব সংস্কৃতিকে নতুন কালের শহুরে সভ্যতার গ্রাস থেকে রক্ষা করার আপ্রাণ চেষ্টা করেও শেষ পর্যন্ত সে হয়েছে এক পরাজিত সৈনিক। তাই উপন্যাস্টির আবেদন অন্য যাই থাক, কাহারদের নিজস্বতাকে রক্ষার জন্য বনোয়ারীর সংগ্রাম ও ব্যর্থতাকে ঘিরে প্রত্যাশা প্রাপ্তির দ্বন্দ্ব জনিত এক করুণ ট্র্যাজিক সুরই উপন্যাসের অন্তিম আবেদন হিসাবে ধরা দিয়েছে। ঠিক একই কথা বলা যায় 'তিতাস একটি নদীর নাম' উপন্যাস প্রসঙ্গেও। মালোদের নিজস্ব সংস্কৃতিকে রক্ষা করার জন্য বাসন্তী ও নোহনের সংগ্রাম ও ব্যর্থতাও এক প্রত্যাশা আর প্রাপ্তির দ্বান্দ্বিক সুরকেই আমাদের কাছে তুলে ধরে। মালোদের নিজস্ব সংস্কৃতি বলতে ছিল ভাটয়ালি গান, কীর্তন গান প্রভৃতি লোক সংগীত। কিন্ত কালের স্রোতে বাইরে থেকে আসা চটুল যাত্রাগান সেই সংস্কৃতির ওপর ক্রনশ থাবা বসাতে শুরু করে, তাদের গোষ্ঠীবদ্ধ জীবনের একতাকে ভেঙে দেয়। এই অবস্থায় দলে দলে মালো ছেলেরা যাতাগানের অভিনয়ে নাম লেখায়, নেয়ে পুরুষেরা ভিড় করে সেই নতুন কালের যাত্রা গানের আসরে। চোখের সামনে মালোদের নিজস্ব সংস্কৃতির এই সংকটকে চেয়ে চেয়ে দেখা সম্ভব হয় না বাসন্তী আর নোহনের পক্ষে। তাই প্রাণপণ চেষ্টা করেছে তারা মালোসমাজকে এই অপসংস্কৃতির স্রোতে ভেলে যাবার হাত থেকে রক্ষা করতে। ওদিকে যখন সারা রাত যাত্রা গানের আসর বসেছে মালো পাড়াতেই; তখন নোহনের ঘরে তারা মালোদের নিজস্ব লোকগানের আসর বসিয়ে স্মৃতির ভান্ডার থেকে বাছা বাছা গান পরিবেশন করে মালোদেরকে তাদের ঐতিহ্য স্মরণ করিয়ে দিতে চেয়েছে। প্রাথমিক ভাবে তারা সফলও হয়েছে; কিন্ত শেষ রক্ষা আর হয়ে ওঠে না। আবার বাঁধ ভাঙা জলের মতোই দলে দলে মালো নারী পুরুষ সেই যাত্রার আসরের দিকেই ঝুঁকেছে। বাসন্তী ও মোহনের মিলিত সংগ্রাম পারেনি মালো সংস্কৃতির এই ভাঙনকে রুখতে। তাই বনোয়ারীর মতোই মালো সংস্কৃতির ধারক ও বাহক এই দুই সৈনিকের ব্যর্থতাকে ঘিরে তাদের অস্তিত্ব জুড়ে শুধুই যেন জেগে থেকেছে এক প্রত্যাশা ও প্রাপ্তির দ্বন্দ্ব। যার পরিচয় ঔপন্যাসিক মাত্র তিনটি বাক্যে ফুটিয়ে তুলেছেন। দলে দলে মালোরা যখন কালোবরণে বাড়ির যাত্রার আসরে দিকে চলেছে, তখন------

"মাত্র দুইটি নরনারী গেল না। তারা সুবলার বউ আর নোহন। অপমানে সুবলার বউ বিছানায় পড়িয়া রহিল, আর বড় দুঃখে নোহলের দুই চোখ ফাটিয়া জল আসিতে লাগিল।"১৩

সর্বোপরি উপন্যালের শেষে মালোদের গোষ্ঠীজীবনের প্রত্যাশা ও প্রাপ্তির দ্বন্ব আরেক মাত্রা পেয়েছে তিতাসের জল শুকিয়ে গিয়ে তার বুকে চর জেগে ওঠার ঘটনাকে ঘিরে। আমাদের মনে হয়, তিতাস নির্ভর মালোসম্প্রদায়ের জীবন নিয়ে লেখা এই' উপন্যাসের অন্যতন মূল সংকট, প্রত্যাশা প্রাপ্তির মূল বিরোধ এখানেই। আমরা দেখেছি তিতাসের উপর মালোদের নির্ভরতা কতখানি, বিশেষত উপন্যাসের প্রথম খন্ডের 'তিতাস একটি নদীর নাম' অধ্যায়েই তার পরিচয় দিয়েছেন ঔপন্যাসিক। মায়ের মতোই তিতাসের উপর মালোদের নির্ভরশীলতা। তাই তিতাস নদীতে মাছ ধরতে যাওয়া সন্তান কিংবা স্বামীর জন্য দুশ্চিন্তা করেনা কোন মালো জননী কিংবা মালোদের বউ। কারণ তিতাস মায়ের মতোই তাদের রক্ষা করবে বলে তাদের বিশ্বাস। শুধু তাই নয়, এতো গেল বাড়ির নেয়েদের তিতাসের উপর নির্ভরশীলতার কথা। অন্যদিকে মায়ের মতোই তিতাস মালোদের মুখে অন্নও তুলে ধরে। কারণ তিতাস থেকে মাছ ধরে জীবিকা নির্বাহ করে মালো সম্প্রদায়। তাই মালো পুরুষদের তিতাসকে নিয়ে গর্বের শেষ নেই। বিজয় নানের আরেকটি যে নদীর কথা এই অধ্যায়ে আছে, সেই বিজয় কখনো কখনো শুকিয়ে গিয়ে তার উপর নির্ভরশীল জেলে সম্প্রদায়ের জীবনকে অতিষ্ঠ করে তোলে----- 
11 | তিতাস একটি নদীর নাম' : ব্যক্তিজীবন বনাম গোষ্ঠীজীবনের প্রত্যাশা ও প্রাপ্তির দ্বন্দ্ব

" সে সব গাঁয়ে তারা দেখিয়াছে, চৈত্রের খরায় নদী কত নিষ্করুণ হয়। একদিক দিয়া জল শুখায় আর একদিক দিয়া মাছেরা দমবন্ধ হবার আশঙ্কায় নাক জাগাইয়া হাঁপায়। মাছেদের মত জেলেদেরও তখন দমবন্ধ হইতে থাকে"। 8

কিন্ত তিতাস কখনোই বিজয় নদীর মতো একেবারে নিঃস্ব হয়ে গিয়ে তার উপর নির্ভরশীল মালোদের জীবনকে সংকটের মুখোমুখি ঠেলে দেয়নি। তিতাসের বুকে অনেক জল, তিতাসের তীরের মালোরা তাই বড় বড় জাল ফেলে কত রকনের মাছ ধরতে পারে।যেখানে বিজয়ের বুকে তিন কোণা ঠেলা জালই ঠিক মতো ডোবে না(যে যে জালকে তীতাসের পারের মালোরা জাল বলেই মনে করে না)। ফলে বিজয়ের তীরের মালোদের মতো তাদেরকে ঠেলা জাল ঘাড়ে করে গ্রাম গ্রামান্তরে খানা ডোবা খুঁজে দু আনা আর দশ পয়সার নোরলা ধরার জন্য হন্যে হয়ে ঘুরতে হয় না।তিতাস তার সন্তানদের কখনো এমন কঠিন সংকটের মধ্যে ফেলেনি।তাই তিতাসের তীরের মালোদেরকে বিজয়ের তীরের জেলেদের সম্পর্কে দুঃখ করে বলতে শোনা যায় ---

"বিজনার পারের মালোগুষ্ঠি বড় অভাগা রে ভাই, বড় অভাগা।" ১৫

কিন্ত বিজয়ের তীরের জেলেদের জীবনের দুর্ভাগ্য যে তাদের জীবনের ক্ষেত্রেও সত্য হয়ে উঠবে, একথা তিতাস পাড়ের মালোরা স্বপ্নেও যেন কল্পনা করেনি। উপন্যাসের শেষে সেই তিতাসের জল শুকিয়ে গিয়ে তার বুকে চর জেগে ওঠার দৃশ্যটি যেন মালোদের কাছে এক দুঃস্বপ্নের রাত্রির মতোই মনে হয়েছে।কারণ মালোরা চাষের কাজ জানে না, জলই তাদের জীবন। আর তাই সেই জল শুকিয়ে যাবার অর্থ তাদের জীবনের বাঁচার একমাত্র পথটিই বন্ধ হয়ে যাওয়া।তাই সমগ্র উপন্যাস জুড়ে তিতাসকে ঘিরে মালোদের যে প্রত্যাশা, তা যেন উপন্যাসের শেষে এক চূড়ান্ত সংকটেরই মুখোমুখি দাঁড়িয়েছ। কালের স্রোতই এখানে যেন নিষ্sুর নিয়তির রূপ নিয়ে সত্য হয়ে উঠেছে মালোদের জীবনে। অধ্যাপক অচিন্ত্য বিশ্বাসের ভাষায়-----

"আশ্র:বাস, আস্থা, মাতৃত্ব, শান্তি ও স্বপ্নের যে নীড় তিতাসের প্রারম্ভিক খন্ডে গড়ে তোলা হয়, সেই নীড়ই তো উপন্যালের অন্তিনে পোঁছে খান খান হয়ে ভেঙে যায়। তখন জলের বদলে জেগে থাকে তৃষ্ণা, স্বপ্নের বদলে জাগে অন্ধকার।"১৬

উপন্যাসে শেষ পর্যায়ে তিতাসের নিঃস্ব হয়ে ওঠা বা তার বুকে তৃষ্ণা জেগে থাকা তো শুধু মাত্র তিতাসের উপর নির্ভরশীল মালোসম্প্রদায়ের অর্থনৈতিক নিরাপত্তার বিপন্নতাকে চিহ্নিত করেনা; বরং তিতাসকে নিয়ে সমগ্র উপন্যালে মালোদের যে গর্ব, যে নির্ভরশীলতা, যে প্রত্যাশা-----সেই প্রত্যাশারই বিপন্নতাকে চিহ্নিত করে দেয়।

কাজেই একথা বলাই যায় যে, অদ্বৈত মল্লবর্মণ তাঁর 'তিতাস একটি নদীর নাম' উপন্যাসে মালোজীবনের আধারে মানব জীবনের প্রত্যাশা ও প্রাপ্তির চিরকালীন দ্বন্দ্বটিকেই যেন রূপ দিতে চেয়েছেন। মাণিক বন্দ্যোপাধ্যায় তাঁর 'পুতুল নাচের ইতিকথা' উপন্যালে দেখিয়েছেন মানব জীবনে নিয়তির ভূমিকাকে। মানুষ চায় এক , আর হয় আর এক। কোন এক অদৃশ্য শক্তির অঙ্গুলি হেলনে যেন আমাদের জীবন নিয়ন্ত্রিত হয়, আমরা নিজেদের ইচ্ছা মতো জীবনকে পরিচালিত করতে পারিনা। জীবন রঙ্গভূমিতে আমরা প্রত্যেকেই যেন এক একটা পুতুল, যাকে আড়াল থেকে নিজের মতো করে চালনা করে অন্য একজন। তাকে নিয়তিই হয়তো নাম দেওয়া চলে। ঠিক একই কথা যেন বলা চলে অদ্বৈত মল্লবর্মণের 'তিতাস একটি নদীর নাম' উপন্যাস প্রসঙ্গেও। এই উপন্যাসের বেশিরভাগ চরিত্র, সম্পর্ককে ঘিরে তৈরি হয়েছে একধরনের অতৃপ্তি------- 
অপূর্ণতার আখ্যান। উপন্যাসের শেষে তিতাসের শুকিয়ে যাওয়া যেমন একদিকে মালোদের আশা আকাঙ্খার সংকট সৃষ্টি করেছে, তৈরি করেছে তাদের অর্থনৈতিক নিরাপত্তার সংকট-----তেমনি তিতাসের এই শুকিয়ে গিয়ে নিঃস্ব হয়ে ওঠার আর এক প্রতীকি তাৎপর্যও যেন আমরা উপলধ্ধি করতে পারি। বস্তুত তিতাসের সাথে মালোদের জীবনের এক অদ্ভুত মিল দেখিয়েছেন অদ্বৈত মল্লবর্মণ। তিতাসের বুকে যেমন একদিন অনেক জল ছিল কিন্ত শেষপর্যন্ত তা শুকিয়ে গিয়ে শুধুই তৃষ্ণাকে জাগিয়ে রাখে; প্রত্যেক মালো নরনারীর জীবনেও ছিল অনেক আশাআকাজ্খা, অনেক প্রত্যাশা-----যা তিতাসের জলের মতোই শেষপর্যন্ত শুকিয়ে গিয়ে জেগে থেকেছে শুধুই তৃষ্ণা।আসলে তিতাস এখানে মালোদের ভাগ্যেরই প্রতীক স্বরূপ। তিতালের বুকে আজ যে শূন্যতা, তা তো প্রত্যেক মালো নরনারীর জীবনেই আমরা লক্ষ করেছি এবং যা আমাদের প্রবন্ধের আলোচনার বিষয়ও বটে। উপনাসটির প্লটের দিকে তাকালে দেখা যায়, এই উপন্যাটি আর পাঁচটা উপন্যাসের থেকে কিছু স্বতন্ত্র।এই উপন্যাসে চারটি খন্ড আছে এবং প্রতিটি খন্ডে আবার দুটি করে অধ্যায়। আর এই অধ্যায়গুলিও যেন স্বতন্ত্র এক একটি উপন্যাসের মতো, যাদের মধ্যে আপাতভাবে যেন কোন সংযোগ নেই বলে মনে হয়। কিন্তু জীবনের প্রত্যাশা প্রাপ্তির যে চিরন্তন দ্বন্দ্বটিকে ঔপন্যাসিক বিভিন্ন মালো নর-নারীর জীবনে দেখিয়েছেন, সেই দ্বন্বজনিত ট্র্যাজিক সুরই যেন উপন্যাসটির আপাত বিচ্ছিন্ন কাহিনিধারা ও আপাত সম্পর্কহীন চরিত্রগুলিকে এক সূত্রে ব্ঁেধে দিয়েছে।কারণ উপন্যাসটির প্রায় প্রত্যেকটি চরিত্রই দুর্ভাগ্যের শিকার, প্রত্যেকেরই অস্তিত্ব জুড়ে চাওয়া পাওয়ার দ্বন্দ্বটিকেই বড় হয়ে উঠতে আমরা দেখি। ফলে এখানেই উপন্যাসটির প্লটের বিশিষ্টতা। তবে আগেই আলোচনা সূত্রে আমরা বলেছি, এই উপন্যাস ব্যক্তি মালোজীবনের কথা নয়। শেষপর্যন্ত উপন্যাসটি ব্যক্তিজীবনকে অতিক্রেন করে সমগ্র মালোজীবনের তথা সমষ্টি জীবনেরই প্রত্যাশা আর প্রাপ্তির দ্বন্দকে তুলে ধরেছে। আর মালোদের সংস্কৃতির অবক্ষয় ও তিতাসের বুকের জল শুকিয়ে যাওয়ার প্রসঙ্গকে ঘিরেই তৈরি হয়েছে এই দ্বন্দ্ব। এখানেই উপন্যাসটির বিশিষ্টতা।তাই 'তিতাস একটি নদীর নাম' যেন মানব জীবনের চিরকালীন প্রত্যাশা ও প্রাপ্তির দ্বন্বেরই আরেক নাম।সে দ্বন্দ্ব যেমন ব্যক্তি জীবনের, তেমনি গোষ্ঠী জীবনেরও বটে।

তথ্যসূত্রः

১।সরোজ বন্দ্যোপাধ্যায়ঃ'বাংলা উপন্যাসের কালান্তর',দেজ পাব্লিশিং, পঞ্চম সংস্করণঃনভেম্বর ২০০৩, কার্তিক ১৪১০, পৃ.৩২২

২।অদ্বৈত মল্লবর্মণঃ'তিতাস একটি নদীর নাম', মাইতি বুক হাউস, কলকাতা-৭৩,প্রথম সংং্করণঃজানুয়ারি ২০১৪, পৃ.১২২ ৩। ডঃ হীরেন চটোপাধ্যায়(সম্পাদনা):'তিতাস একটি নদীর নামঃ উপন্যাসেরও', 'তিতাসের অনান্নী অঙ্গনা' নামক প্রবন্ধ, বঙ্গীয় সাহিত্য সংসদ, প্রথম প্রকাশঃরাস পূর্ণিনা ১৪০৭, পৃ.১৭৪

৪।২।অদ্বৈত মল্লবর্মণঃ'তিতাস একটি নদীর নাম', মাইতি বুক হাউস, কলকাতা-৭৩,প্রথম সংস্করণঃজানুয়ারি ২०১8, পৃ.১১৮

৫।ळ,পৃ.৫৩

৬।ঝ, পৃ.১২৩ 
13 | তিতাস একটি নদীর নাম' : ব্যক্তিজীবন বনাম গোষ্ঠীজীবনের প্রত্যাশা ও প্রাপ্তির দ্বন্দ্ব

৭। ডঃ হীরেন চটোপাধ্যায়(সম্পাদনা): 'তিতাস একটি নদীর নামঃ উপন্যালেরও','তিতাস একটি নদীর নামঃপ্লটের বিচিত্র বিন্যাস' নামক প্রবন্ধ, বঙ্গীয় সাহিত্য সংসদ, প্রথম প্রকাশঃরাস পূর্ণিমা ১৪০৭, পৃ.১৯১

৮।অদ্বৈত মল্লবর্মণঃ'তিতাস একটি নদীর নাম', মাইতি বুক হাউস, কলকাতা-৭৩, প্রথম সংস্করণঃজানুয়ারি ২০১৪, পৃ.২২৪

৯।ঐ,পৃ.২২৪

১০।ঐ,পৃ.২২৯

১১।ঐ,পৃ.২৩০

১২।ঐ,পৃ.২৫৭

১৩।ঐ,পৃ.২৪৮

১৪।ঐ,পৃ.৫

১৫।ঐ, भৃ.৭

১৬।৭। ডঃ হীরেন চট্টোপাধ্যায়(সম্পাদনা):'তিতাস একটি নদীর নামঃ উপন্যাসেরও','তিতাস একটি নদীর নামঃপ্লটের

বিচিত্র বিন্যাস' নামক প্রবন্ধ, বঙ্গীয় সাহিত্য সংসদ, প্রথম প্রকাশঃরাস পূর্ণিনা ১৪০৭, পৃ. ১৯০ 